\title{
Experimental study of USPIO-enhanced MRI in the detection of atherosclerotic plaque and the intervention of atorvastatin
}

\author{
TING SHA $^{1 *}, \mathrm{CHUNMEI}^{2^{*}}{ }^{*}, \mathrm{WEI} \mathrm{FU}^{1 *}, \mathrm{JI} \mathrm{HAO}^{2}, \mathrm{LEI} \mathrm{GONG}^{2}, \mathrm{HAO} \mathrm{WU}^{2}$ and QINGDUI ZHANG $^{2}$ \\ ${ }^{1}$ Institute of Cardiovascular Diseases, Xuzhou Medical College, Xuzhou, Jiangsu 221002; \\ ${ }^{2}$ The Second Affiliated Hospital, Xuzhou Medical College, Xuzhou, Jiangsu 221000, P.R. China
}

Received December 23, 2015; Accepted April 18, 2016

DOI: 10.3892/etm.2016.3266

\begin{abstract}
Ultrasmall superparamagnetic iron oxide (USPIO) can identify atherosclerotic vulnerable plaque and atorvastatin can stabilize vulnerable plaque by inhibiting the inflammatory response. Using balloon injury in rabbit abdominal aortic endothelial cells and p53 gene transfecting the local plaque, we established an atherosclerotic vulnerable plaque model. In the treatment group, animals were treated with atorvastatin for 8 weeks. At the end of week 16, the animals in each group underwent medication trigger. USPIO-enhanced MRI was utilized to detect vulnerable plaque formation and the transformation of stable plaque in the treatment group. Pathological and serological studies were conducted in animal sera and tissues. The images from the USPIO-enhanced MRI, and the vulnerable plaque showed low signal, especially on $\mathrm{T} 22^{*}$-weighted sequences $\left(\mathrm{T} 2{ }^{*} \mathrm{WI}\right)$. Plaque signal strength reached a negative enhancement peak at $96 \mathrm{~h}$. Compared with the other groups, lipids, cell adhesion molecule-1 and vascular cell adhesion molecule-1 levels were significantly lower $(\mathrm{P}<0.05)$ in the treatment group. In conclusion, USPIO-enhanced MRI can identify vulnerable plaque formation by deposition in macrophages, while atorvastatin is able to inhibit the progression of atherosclerosis and promote plaque transformation to the stable form.
\end{abstract}

\section{Introduction}

Atherosclerosis (AS) is a common pathological basis of many cardiovascular and cerebrovascular diseases. It is the most common disease among cardiovascular diseases that may be life threatening. The prevailing view is that the occurrence and development of AS is a chronic inflammatory process $(1,2)$. Endothelial cells synthesize a variety of adhesion molecules

Correspondence to: Dr Chunmei Qi, The Second Affiliated Hospital, Xuzhou Medical College, Xuzhou, Jiangsu 221000, P.R. China

E-mail: qsk608@163.com

"Contributed equally

Key words: superparamagnetic iron oxide, atherosclerotic plaque, cell adhesion molecule-1 in response to the injury factors. The mononuclear cells adhere to the vessel wall, enter through the endothelial cells and differentiate into macrophages. Subsequently, mediated by scavenger receptor A (SRA), the macrophages engulf a large amount of lipid deposited in the intimal, and gradually transform into foam cells (3).

The concentration of inflammatory mediators in the local environment increase vascular damage, accompanied by the change of activities of the local coagulation, complement, and kinin-kallikrein systems (4). The change of local signal microenvironment leads to smooth muscle cells and endothelial cell dysplasia into the vessel wall. Part of smooth muscle cells secret collagen fibers, elastic fibers and proteoglycans, which embed lipid and cells forming a fibrous plaque (5). The further involvement of inflammatory cells and inflammatory factors leads to the fibrous plaque cap rupturing and shedding, resulting in the exposure of deep lesions (collagen, tissue necrosis disintegrating substance, cholesterol and calcium). The exposure leads to the formation of thrombosis and vascular embolism and triggers serious cardiovascular and cerebrovascular events (3).

Previous findings have confirmed that macrophages are important in the atherosclerotic vulnerable plaque formation process. Ultrasmall superparamagnetic iron oxide (USPIO) can be engulfed by macrophages and as a result, engulfed USPIO may deposit within macrophages (6-8). The engulfed USPIO inside macrophages can be detected using Prussian blue staining. According to this feature, USPIO-enhanced MRI becomes an effective technique in detecting AS vulnerable plaque (9). It has been demonstrated that the expression of cell adhesion molecule 1 (ICAM-1) and vascular cell adhesion molecule-1 (VCAM-1) in macrophages increase in atherosclerotic rabbits (10). Adhesion molecules are important inflammatory factors in the plaque formation process. ICAM-1 and VCAM-1 are cell surface transmembrane glycoproteins that regulate the adhesions and interactions between cells, as well as between cells and matrix. They promote the adhesion of leukocytes to endothelial cells, which is the initiating step during the AS process (11). Previous experiments have shown that statins have lipid-lowering, anti-inflammatory, and plaque stability, albeit the mechanism is not clear. The rabbit was selected to establish the AS model, given atorvastatin pharmaceutical intervention, and related indicators were measured to verify the role of atorvastatin in the vulnerable plaque. 


\section{Materials and methods}

Experimental animals and groups. In the present study, 30 healthy male New Zealand (NZ) white rabbits (Experimental Animal Center of Xuzhou Medical College, Xuzhou, China), weighing 2.5-3.0 kg were used. The rabbits were kept in single cages, with access to water and feed ad libitum. The animals were divided into three groups: group A, high-fat ( $1 \%$ cholesterol, $0.2 \%$ bile salt, $10 \%$ lard, $88.8 \%$ normal diet, each $120-140 \mathrm{~g} /$ day) feeding alone group; group B, balloon-induced injury plus high cholesterol feeding group; and group $\mathrm{C}$, atorvastatin group ( $\mathrm{n}=10 /$ group). Approval for the animal studies was provided by the Xuzhou Medical Ethics Committee, license number: CMCACUC2009-04-135.

Atherosclerotic vulnerable plaque model. In total, 30 male NZ white rabbits were fed with a high-fat diet. The rabbits in groups $\mathrm{B}$ and $\mathrm{C}$ underwent abdominal aorta balloon-induced injury after one week. Briefly, the rabbits were anesthetized through with $30 \mathrm{mg} / \mathrm{kg}$ sodium pentobarbital via injecting the rabbit ear vein. Puncture of the right femoral artery was performed and a balloon catheter of $3.5-\mathrm{mm}$ diameter and $15 \mathrm{~mm}$ in length was introduced into the aorta approximately $20 \mathrm{~cm}$ along the 0.014 inch guide wire. The balloon was inflated by injecting distilled water up to 8 atmospheres and was then pulled back to the common iliac artery. The balloon was repeatedly pulled back 3 times to injure the abdominal aortic endothelium. The femoral artery was ligated and intramuscular injection of penicillin was given to prevent infection. A high-cholesterol diet ( $1 \%$ cholesterol, $0.2 \%$ bile salt, $10 \%$ lard, and $88.8 \%$ normal diet, each 120-140 g/day) was provided to experimental animals for 8 weeks.

Wild-type Ad-p53 virus transfection in local plaque. At the end of week 8, the rabbits underwent Ad-p53 transfection. The animals were anesthetized through the injection of $30 \mathrm{mg} / \mathrm{kg}$ sodium pentobarbital via the rabbit ear vein. An incision was made to the abdominal cavity to expose the abdominal aorta to locate the largest patch at the vessel segment between the right renal artery and common iliac artery; this was the site where the viral vector were transfected. Following transient closure of the blood vessel in the two sides, $10 \mu 1$ of $1.5 \times 10^{10} \mathrm{pfu} / \mathrm{ml}$ Ad5-p53 recombinant vector were injected into the plaque. After $10 \mathrm{~min}$, the blood supply was restored in the abdominal artery. A suture mark was added in the ligation site and the abdominal cavity was closed. Penicillin was intramuscularly injected to prevent infection.

First MRI examination to detect plaque formation. At the end of week 8, an MRI (Philips MedicalSystem, Eindhoven, The Netherlands) examination was conducted on all the experimental rabbits to monitor whether atherosclerotic plaques were formed. Various plaque characteristics were observed and recorded. At the end of week 8 , when plaque formation was confirmed by MRI, the drug intervention $(2 \mathrm{mg} / \mathrm{kg} / \mathrm{day})$ was given immediately for 8 weeks. At the end of week 16, based on the methodology previously described (12), peritoneal injection of $0.15 \mathrm{mg} / \mathrm{kg}$ Chinese Russell's viper venom (CRVV Guangdong, Research Institute of Snake Venom, Guangdong, China) was performed twice. After $30 \mathrm{~min}$ and
$24 \mathrm{~h}, 0.02 \mathrm{mg} / \mathrm{kg}$ histamine (Dongfeng Biological Technology, Shanghai, China) was injected via the ear vein.

Second MRI examination to detect breakage of plaque. Aortic MRI imaging was conducted at time points prior to medication and $0,24,48,72$ and $96 \mathrm{~h}$ after the medication. The signal intensity (SI), signal-to-noise ratio (SNR), size of lumen area and size of plaque area were determined to compare difference in plaque characteristics in the three groups.

Blood biochemical indices and detection of inflammatory factors. Total cholesterol (TC), triglyceride (TG), high-density lipoprotein cholesterol (HDL-C) and low-density lipoprotein cholesterol (LDL-C) levels were measured in all the rabbits prior to and following the intervention of simvastatin. The blood was collected in the morning on fasting via the marginal ear vein. VCAM-1 and ICAM-1 levels were determined using enzyme-linked immunosorbent assay (ELISA).

Histopathological detection. Morphological characteristics of ruptured and unruptured plaque were observed using hematoxylin and eosin (H\&E) and Masson's trichrome staining. The deposition of the USPIO particles within the plaque was observed using Prussian blue staining.

Electron microscopy measurement. The ultrastructural changes and apoptosis of cells were observed using transmission electron microscope (TEM).

Statistical analysis. Quantitative data were presented as the mean $\pm \mathrm{SD}$, of multiple sets of variables and compared using one-way ANOVA and Cochran's Q test pairwise comparisons, $\mathrm{P}<0.05$ was considered statistically significant differences. Data were analyzed using SPSS 16.0 software.

\section{Results}

Comparison of blood lipid profile. TC, $\mathrm{LDH}$, and triglycerol blood levels were significantly higher $(\mathrm{P}<0.05)$ at the end of week 16 in all three groups. At the end of week 16, the inter-group comparison showed a significant reduction in blood $\mathrm{TC}, \mathrm{LDH}$, and triglycerol of group $\mathrm{C}(\mathrm{P}<0.05)$ in comparison to groups A and B (Table I).

Comparison of the expression of VCAM-1 and ICAM-1. VCAM-1 and ICAM-1 levels were significantly higher $(\mathrm{P}<0.05)$ at the end of week 16 in groups A and B. Inter-group comparison at the end of week 16 showed that VCAM-1 and ICAM-1 levels were significantly lower $(\mathrm{P}<0.05)$ as compared to groups A and B (Table II).

Comparison of MRI indices. Indices in group B or C were statistically different from the counterparts in group A regarding EEMA, LA, PA, LAS\%, VD, LD, PT, and EI values (Table III).

Comparison of MRI imaging among the different groups. In group A, a narrow lumen ring is evident in the images as a flator crescent-shaped plaque protruding into the lumen (Fig. 1). The lipid composition basically can be distinguished in terms of the prominent plaque. The fibrous caps were clearly evident 
Table I. Comparison of blood lipid profile.

\begin{tabular}{lccc}
\hline Variables & Group A & Group B & Group C \\
\hline TC (mmol/l) & & & \\
Day 0 & $1.331 \pm 0.069$ & $1.324 \pm 0.054$ & $1.293 \pm 0.047$ \\
End of W16 & $10.911 \pm 0.646^{\mathrm{a}}$ & $11.571 \pm 0.683^{\mathrm{a}}$ & $2.388 \pm 0.128^{\mathrm{ab}}$ \\
LDH (mmol/l) & & & \\
Day 0 & $0.548 \pm 0.042$ & $0.548 \pm 0.042$ & $0.533 \pm 0.048$ \\
End of W16 & $7.159 \pm 0.188^{\mathrm{a}}$ & $8.286 \pm 0.119^{\mathrm{a}}$ & $2.087 \pm 0.056^{\mathrm{b}}$ \\
HDL (mmol/l) & & & \\
Day 0 & $1.046 \pm 0.062$ & $1.128 \pm 0.051$ & $1.286 \pm 0.119$ \\
End of W16 & $0.690 \pm 0.045$ & $0.708 \pm 0.030$ & $0.699 \pm 0.021$ \\
Triglycerol & & & \\
(mmol/l) & & & \\
Day 0 & $1.079 \pm 0.068$ & $1.081 \pm 0.041$ & $1.113 \pm 0.047$ \\
End of W16 & $1.24 \pm 0.09^{\mathrm{a}}$ & $4.977 \pm 0.121^{\mathrm{a}}$ & $2.106 \pm 0.073^{\mathrm{ab}}$ \\
\hline
\end{tabular}

${ }^{\mathrm{a}}$ For groups $\mathrm{A}$ and $\mathrm{B}$ at week 16 each index was statistically significant compared with 0 weeks, $\mathrm{P}<0.05$. ${ }^{\mathrm{b}}$ For groups $\mathrm{A}, \mathrm{B}$ and $\mathrm{C}$, the index was compared and found to be statistically significant at the end of week 16 , $\mathrm{P}<0.05$. TC, total cholesterol, W, week.

Table II. Comparison of VCAM-1 and ICAM-1.

\begin{tabular}{lccc}
\hline Variables & Group A & Group B & Group C \\
\hline VCAM-1 (pg/ml) & & & \\
Day 0 & $1.100 \pm 0.065$ & $1.115 \pm 0.043$ & $1.104 \pm 0.040$ \\
End of W16 & $2.018 \pm 0.062^{\mathrm{a}}$ & $2.477 \pm 0.060^{\mathrm{a}}$ & $1.420 \pm 0.046^{\mathrm{b}}$ \\
ICAM-1 (pg/ml) & & & \\
Day 0 & $0.954 \pm 0.046$ & $0.973 \pm 0.035$ & $0.979 \pm 0.023$ \\
End of W16 & $1.904 \pm 0.033^{\mathrm{a}}$ & $2.920 \pm 0.041^{\mathrm{a}}$ & $1.263 \pm 0.041^{\mathrm{b}}$ \\
\hline
\end{tabular}

${ }^{\mathrm{a} D i f f e r e n c e}$ between day 0 and end of week $16, \mathrm{P}<0.05$. ${ }^{\mathrm{b}}$ Difference between group $\mathrm{C}$ and groups $\mathrm{A}$ and/or $\mathrm{B}$ at the end of week $16, \mathrm{P}<0.05$. VCAM-1, vascular cell adhesion molecule-1; ICAM-1, cell adhesion molecule $1 ; \mathrm{W}$, week.

in some sequences. Lipid composition showed slightly higher signals in T1WI, PDWI and T2*WI as compared to the low signal from the entire plaque in T2WI (Fig. 1). In T2WI, the lipid core cannot be clearly distinguished and the unenhanced T1WI fat-suppression cannot inhibit completely the lipid signal. After injection of USPIO, the high signal in T2*WI was markedly reduced. Pathological results confirmed iron deposition in the high signal area. In other sequences, the changes of plaque signals were not obvious. The sensitivity of USPIO-enhanced sequences did not significantly increase in distinguishing the fibrous caps. In group B, abdominal aortic lumen narrow ring or eccentric plaque was evident in all the scan sequences. The plaque signals were high in T1WI, PDWI and T2*WI and low in T2WI. In the lipid suppression sequence T1WI, the decrease of plaque signal was not obvious. Following the medication trigger, the obvious breakage of plaque was evident. By $96 \mathrm{~h}$ after enhanced USPIO, the plaque signals in the T2WI and T2*WI were significantly reduced. At $96 \mathrm{~h}$ after injection of
Table III. Comparison of indices of MRI $\left(\mathrm{mm}^{2}\right)$.

\begin{tabular}{lrrr}
\hline Variables & Group A & Group A & \multicolumn{1}{c}{ Group A } \\
\hline EEMA $\left(\mathrm{mm}^{2}\right)$ & $12.91 \pm 1.37$ & $21.15 \pm 2.69^{\mathrm{a}, \mathrm{b}}$ & $17.40 \pm 0.58^{\mathrm{a}}$ \\
LA $\left(\mathrm{mm}^{2}\right)$ & $8.71 \pm 0.61$ & $12.49 \pm 0.92^{\mathrm{a}, \mathrm{b}}$ & $10.9 \pm 0.84^{\mathrm{a}}$ \\
LD min $(\mathrm{mm})$ & $1.56 \pm 0.17$ & $2.78 \pm 0.19^{\mathrm{a}, \mathrm{b}}$ & $2.7 \pm 0.23^{\mathrm{a}}$ \\
VD max $(\mathrm{mm})$ & $1.18 \pm 0.08$ & $1.56 \pm 0.19^{\mathrm{a}, \mathrm{b}}$ & $2.36 \pm 0.09^{\mathrm{a}}$ \\
VD min $(\mathrm{mm})$ & $1.06 \pm 0.11$ & $0.74 \pm 0.11^{\mathrm{a}, \mathrm{b}}$ & $2.12 \pm 0.24^{\mathrm{a}}$ \\
PT max $(\mathrm{mm})$ & $0.48 \pm 0.08$ & $0.76 \pm 0.05^{\mathrm{a}, \mathrm{b}}$ & $0.30 \pm 0.07^{\mathrm{a}}$ \\
PT min $(\mathrm{mm})$ & $0.34 \pm 0.05$ & $0.32 \pm 0.04^{\mathrm{a}, \mathrm{b}}$ & $0.20 \pm 0.07^{\mathrm{a}}$ \\
LAS $(\%)$ & $32.15 \pm 5.99$ & $40.40 \pm 6.4^{\mathrm{a}, \mathrm{b}}$ & $37.14 \pm 4.50^{\mathrm{a}}$ \\
EI & $0.32 \pm 0.08$ & $0.55 \pm 0.08^{\mathrm{a}, \mathrm{b}}$ & $0.35 \pm 0.09^{\mathrm{a}}$ \\
\end{tabular}

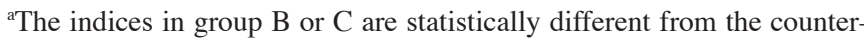
parts in group $\mathrm{A} . \mathrm{P}<0.05$. ${ }^{\mathrm{b}}$ The indices in group $\mathrm{B}$ are statistically different from the counterparts in group C. $\mathrm{P}<0.05$.

contrast, the negative reinforcement reached its peak (Fig. 1). In group $\mathrm{C}$, the reduction of plaques after medication intervention and increase of lumen diameter were observed. Obvious breakage of plaques was not observed (Fig. 1).

Comparison of SNR. A comparison of the SNR change prior to its enhancement at $96 \mathrm{~h}$ and after enhancement revealed that the negative enhancement of plaque signals in group B peaked. By contrast, the changes in groups $\mathrm{A}$ and $\mathrm{C}$ showed no statistical difference $(\mathrm{P}>0.05)$, indicating that the nature and composition of plaques did not change significantly (Fig. 2).

Comparison of microscopic observations in different groups. Comparisons of foam cells, degree of narrowing and existence of plaque hemorrhage were evaluated using $H \& E$ staining. We found some wall thickening in group A and obvious thickening with substantial foam cell formation and large lipid core plaque hemorrhage in group B. Additionally, the wall was relatively smooth and obvious foam cell formation or plaque hemorrhage was not observed (Fig. 3). Moreover, Masson's staining reflected the collagen fiber hyperplasia in the vessel wall. In group B, substantial collagen fiber proliferation was evident (Fig. 4). In addition, Prussian blue staining in group B indicated the iron deposition inside macrophages and no iron deposition was evident in the remaining two groups (Fig. 5). In group B visible lysosome increased, foam cells proliferated, intracellular lipid droplets were visible, while fewer foam cells were present in group $\mathrm{C}$, no lysosomal generation, and iron particles were rare (Fig. 6).

\section{Discussion}

ACS is one of the common diseases of the cardiovascular system forming the basis of the pathophysiology of atherosclerotic plaque, and plaque instability or rupture are clinically acute syndromes and the main reasons especially for acute coronary syndrome $(13,14)$. The instability is caused by multiple factors plaques, including the size of the plaque itself, and thickness of the fibrous cap, apoptosis, the release of various cytokines and enzymes (15), and atherosclerotic plaques block instability and rupture caused by a series of cardiovascular events. 


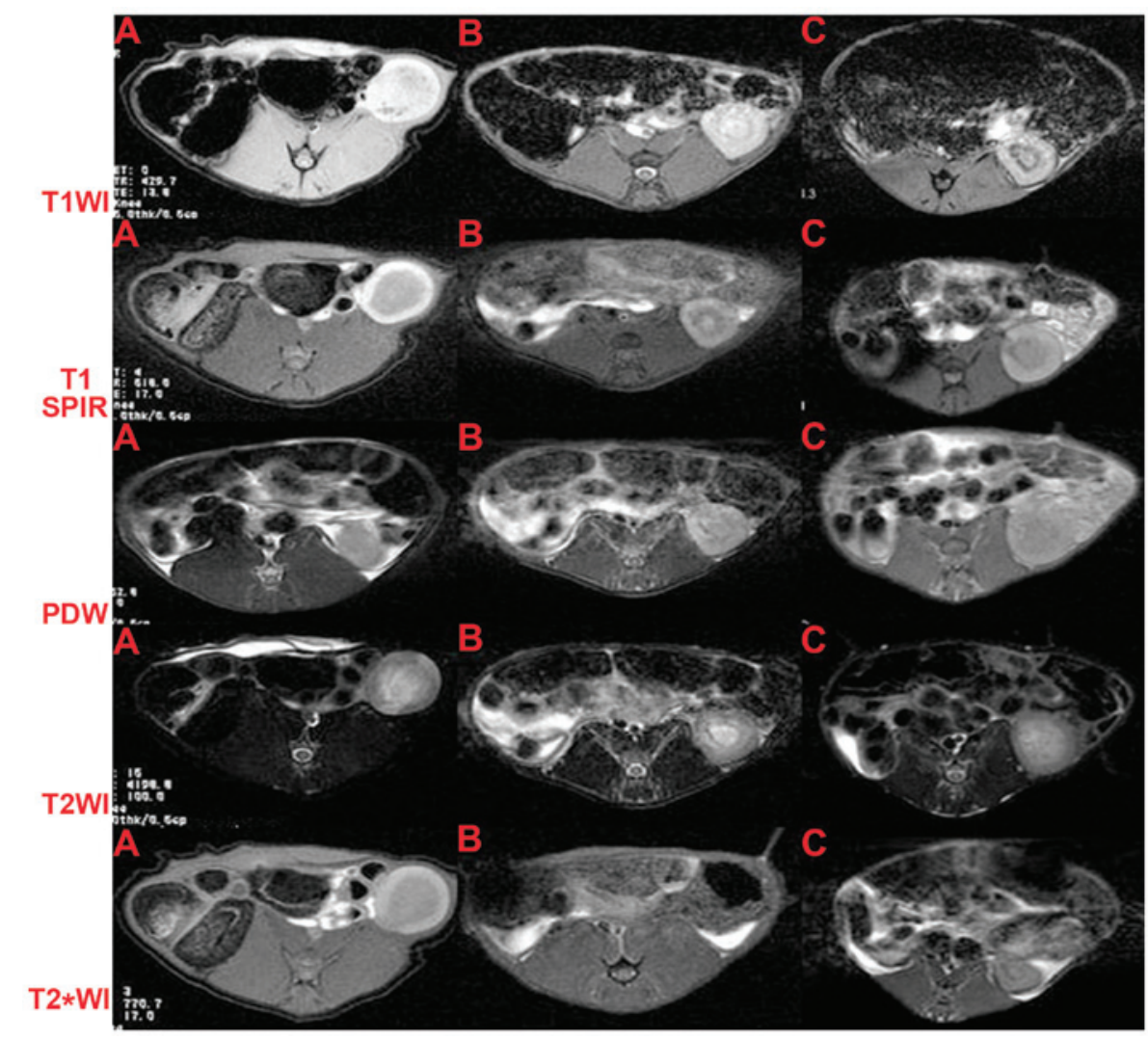

Figure 1. The intensity change of plaque signals in 3 groups after USPIO particle enhancement. In group A, a narrow lumen ring is evident. In group B, abdominal aortic lumen narrow ring or eccentric plaque was evident in all the scan sequences and in group $\mathrm{C}$, the reduction of plaques after medication intervention and increase of lumen diameter were observed. USPIO, ultrasmall superparamagnetic iron oxide.

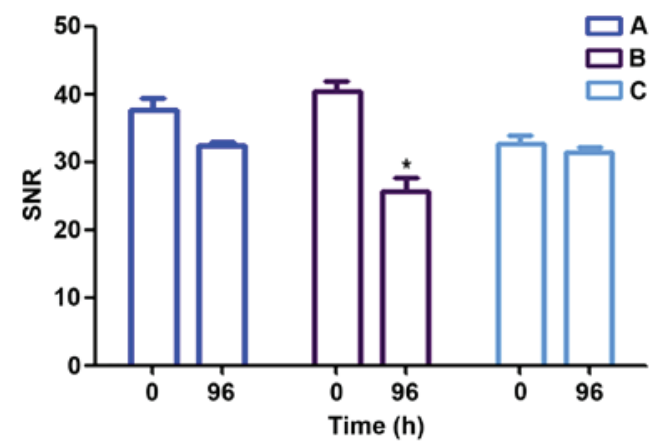

Figure 2. Comparison of SNR. Comparison of the SNR change before its enhancement at $96 \mathrm{~h}$ after enhancement, the negative enhancement of plaque signals in group B peaked. By contrast, the changes in groups A and C showed no statistical difference, indicating that the nature and composition of plaques did not change significantly. SNR, signal-to-noise ratio.

Although the establishment of atherosclerotic vulnerable plaque model has been intensively studied, well-rounded vulnerable plaque models are rare. Some researchers used simple high-fat diet feeding and as a result most of the established models are stable plaque models $(16,17)$. Kurz et al established a model using the Rose Bengal antigen or $\mathrm{Fe}^{3+}$ (18). However, these models were not established based on the disease. A Successfully established animal model of drug-induced plaque breakage using the CRVV and histamine triggering atherosclerotic plaque has been reported (19).

In some studies, the authors found that the regulatory genes of AS plaque apoptosis are mainly c-Myc, $\mathrm{Bcl}-2$ and p53.
Most of p53 genes are mutants, while the expression level of wild-type p53 gene is low. It is considered that abnormal SMC proliferation is related to the gene mutation of p53. However, the roles of wild-type and mutant p53 are different. Wild-type p53 gene plays a role in promoting apoptosis whereas mutant p53 genes inhibit apoptosis. The p53 gene directly transfecting the local plaque and thinning the fibrous cap via the role of pro-apoptosis on VSMC are good methods to establish the animal model of vulnerable plaque. The VSMC apoptotic rate increases and smooth muscle actin-positive cells were significantly reduced after p53 gene transfection, leading to a significantly thinner fibrous cap of the plaque. As a result, the plaque becomes unstable. Chen et al firstly established an animal model of vulnerable plaque using exogenous p53 gene transfecting rabbit AS plaque $(19,20)$. The success rate was significantly increased following a combination of the two methods. In this study, we utilized exogenous p53 gene-transfecting rabbit AS plaque in the abdominal aorta and successfully established a rabbit model of vulnerable plaque.

USPIO is a new type of magnetic MRI contrast agent that is recognized by the reticuloendothelial system (RES) and uptaken by macrophage. Due to the long plasma half-life, USPIO can be widely distributed in the macrophages of RES $(21,22)$. One of the targeting studies of MRI focused on macrophages which reflect the instability of the plaques. Among several methods for the identification, analysis and quantification of the plaque macrophage density, two important types are: i) passive targeting USPIO; and ii) active targeting gadoliniumlabeled antibody particles. The active macrophages inside the 

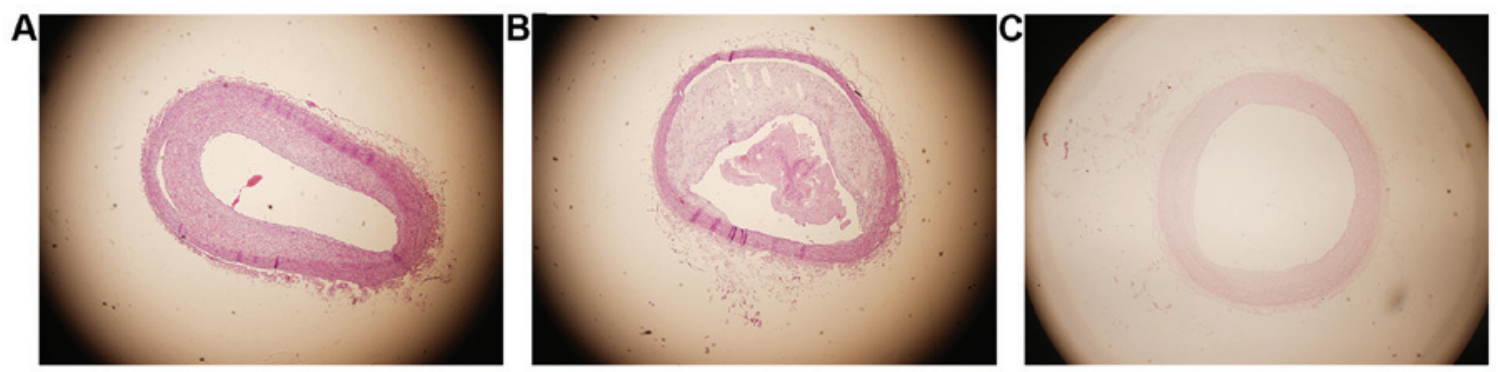

Figure 3. (A) Wall thickening. (B) Significantly reduced lumen. (C) Uniform lumen.
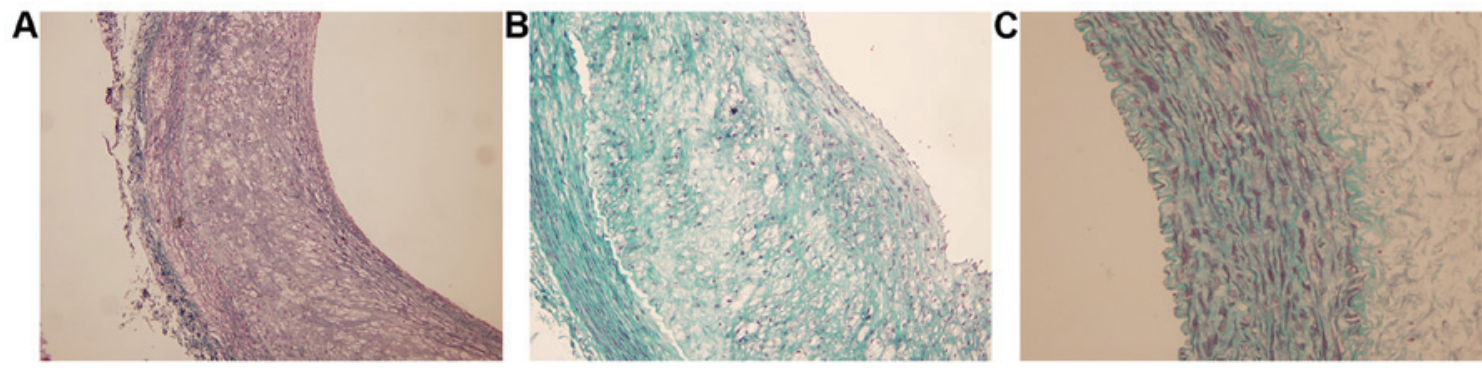

Figure 4. (A-C) Masoon's staining. (B) Substantial foam cell proliferation. The collagen proliferation was not obvious in the 3 groups.
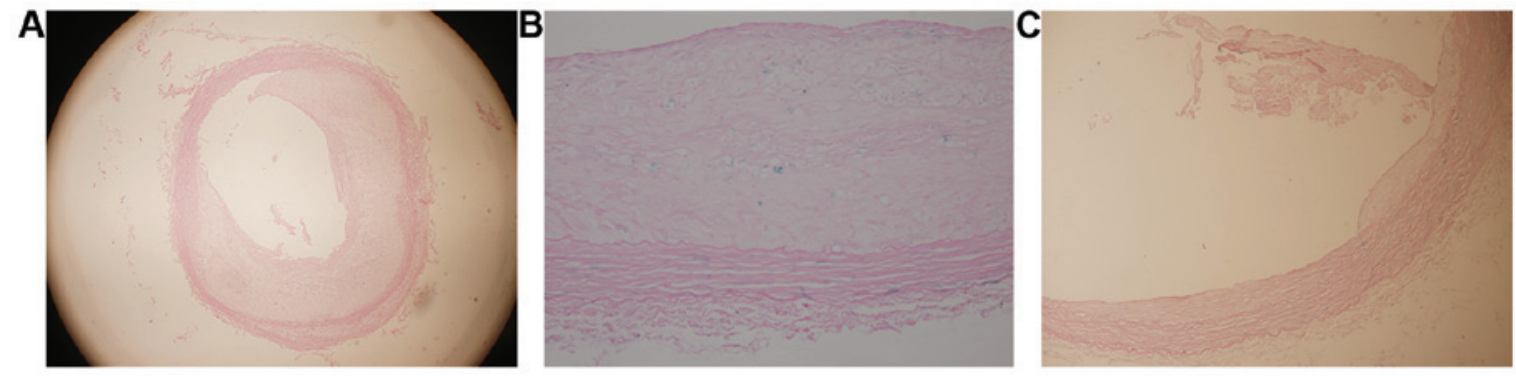

Figure 5. (A) The control group (x100) showed no particle deposition or (C) (x100) visible macrophage USPIO particle deposition, and was (B) the same at high magnification (x200). USPIO, ultrasmall superparamagnetic iron oxide.
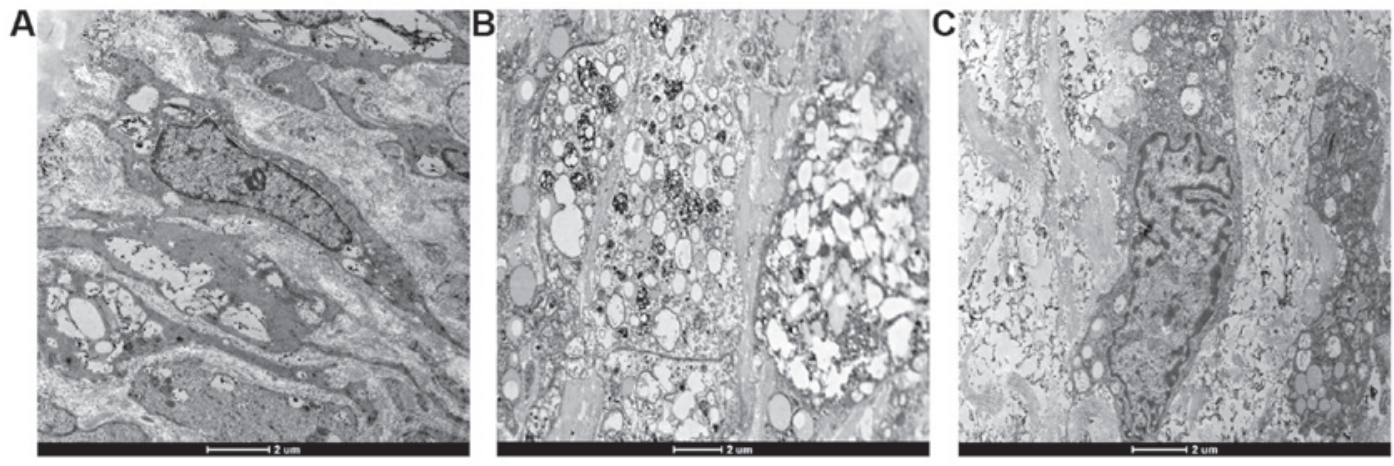

Figure 6. Electron microscopy. (A) Increased lysosomes, (B) foam cell proliferation with visible lipid droplets. (C) Iron particle deposition inside macrophages.

plaque can significantly engulf the iron oxide particles, thus, USPIO-labeled macrophage imaging is often used as a passive target. Nevertheless, the characteristics that macrophages uptake USPIO has been widely confirmed, and the exact route how USPIO enter macrophages remain unclear. When the diameter of iron nanoparticles is less than the critical value of the magnetic particles $(30 \mathrm{~nm})$, it shows superparamagnetism. When it enters the body and binds to plasma protein, these iron nanoparticles are recognized by opsonized RES and engulfed by phagocytes and residue inside the reticuloendothelial cells. The superparamagnetic effect causes an uneven local magnetic field in tissues. When the water molecules diffuse through the 
heterogeneous magnetic field, water accelerates the proton loss phase, and shortens the tissue transverse relaxation time (T2) and longitudinal relaxation time (T1). As a result, the corresponding $\mathrm{T} 1$ and $\mathrm{T} 2$ changes can be detected on MR images. Therefore, USPIO can be used to mark the inflammatory cells and can be observed on MR in vivo. Due to its multi-parameter, multi-sequence characteristics, magnetic resonance can distinguish fat, calcification, fibrous and thrombosis ingredients with a characteristic signal (23).

Effects of statins, other than on lipid modification, have been previously reported (24). Results from clinical trials have shown that statins can significantly reduce cardiovascular-related morbidity and mortality in patients with and without cardiovascular diseases (24). Results from experimental animal models have confirmed that statin therapy can stabilize atherosclerotic plaques, including reducing extracellular lipid deposition, reducing the number of macrophages and the amount of cholesterol inside the intima, increasing collagen and the SMC area, reducing calcification of the intima and angiogenesis, reducing the lipid cores of plaques by reducing LDL levels in the blood, reducing plaque surface tension, strengthening the fibrous cap, and maximally stabilizing and shrinking plaques $(25,26)$. In general, statins have strong anti-proliferation and anti-migration effects. Atorvastatin calcium is a 3-hydroxy-3-methyl-diacyloxy coenzyme (HMA-CoA) reductase inhibitor, by inhibiting HMA-CoA, the synthesis rate-limiting enzyme of TC. Atorvastatin can significantly reduce the blood LDL-C level by inhibiting the synthesis of TC in hepatocytes and upregulating the density and activity of LDL-C receptor on the surface of hepatocytes.

In the present study, we found that VCAM-1 is overexpressed in the AS lesion site. Atorvastatin inhibits VCAM-1 expression and the hyperplasia of the artery intima, leading to the shrinkage of AS plaques. In conclusion, atorvastatin has a role, not only in lowering the lipid profile, but in reversing AS. Inhibiting VCAM-1 expression may be one of the mechanisms in which atorvastatin inhibits AS. We believe that the effective anti-inflammatory dose of atorvastatin may be lower than the effective lipid-lowering doses. The lipid-lowering efficacy is associated with the dose and duration, whereas the anti-inflammatory effect is dose-dependent.

\section{Acknowledgements}

The present study was funded by the Six Talents Peak, no. 2014-WSN-044.

\section{References}

1. Virani SS, Polsani VR and Nambi V: Novel markers of infammation in atherosclerosis. Curr Atheroscler Rep 10: 164-170, 2008

2. Kleemann R, Zadelaar S and Kooistra T: Cytokines and atherosclerosis: A comprehensive review of studies in mice. Cardiovasc Res 79: 360-376, 2008.

3. Wang HD, Yu SQ, Gao F and Qin ML: The anti-atherosclerosiseffect of adiponectin. Chongqing Med J 38: 2509-2510, 2009 (In Chinese).

4. Pasceri V, Willerson JT and Yeh ET: Direct proinflammatory effect of C-reactive protein on human endothelial cells. Circulation 102: 2165-2168 2002.

5. Beck B, Weintraub W and Alexander R: Elevation of C-reactive protein in 'active' coronary artery disease. AMJ Cardiol 65: 168-172, 1990.

6. Ross R: Atherosclerosis - an infammatory disease. N Engl J Med 340: 115-126, 1999.
7. Tang T, Howarth SP, Miller SR, Trivedi R, Graves MJ, King-Im JU, Li ZY, Brown AP, Kirkpatrick PJ, GauntME, et al: Assessment of infammatory burden contralateral to the symptomatic carotid stenosis using high-resolution ultrasmall, superparamagnetic iron oxide-enhanced MRI. Stroke 37: 2266-2270, 2006.

8. Howarth SP, Tang TY, Graves MJ, U-King-Im JM, Li ZY, Walsh SR, Gaunt ME and Gillard JH: Non-invasive MR imaging of infammation in a patient with both asymptomatic carotidatheroma and an abdominal aortic aneurysm: A case report. Ann Surg Innov Res 1: 4, 2007.

9. Metz S, Beer AJ, Settles M, Pelisek J, Botnar RM, Rummeny EJ and Heider P: Characterization of carotid artery plaques with USPIO-enhanced MRI: Assessment of infammation and vascularity as in vivo imaging biomarkers for plaque vulnerability. Int J Cardiovasc Imaging 27: 901-912, 2011.

10. Truskey GA, Herrmann RA, Kait J and Barber KM: Focalincreases in vascular cell adhesion molecule-1 and intimal macrophages at atherosclerosis-susceptible sites in the rabbit aorta after short-term cholesterol feeding. Arterioscler Thromb Vasc Biol 19: 393-401, 1999.

11. Cybulsky MI, Iiyama K, Li H, Zhu S, Chen M, Iiyama M, Davis V, Gutierrez-Ramos JC, Connelly PW and Milstone DS: A major role for VCAM-1, but not ICAM-1, in early atherosclerosis. J Clin Invest 107: 1255-1262, 2001

12. Kolodgie FD, Gold HK, Burke AP, Fowler DR, Kruth HS, Weber DK, Farb A, Guerrero LJ, Hayase M, Kutys R, et al: Intraplaque hemorrhage and progression of coronary atheroma. N Engl J Med 349: 2316-2325, 2003.

13. Danesh J, Wheeler JG, Hirschfield GM, Eda S, Eiriksdottir G, Rumley A, Lowe GD, Pepys MB and Gudnason V: C-reactive protein and other circulating markers of inflammation in the prediction of coronary heart disease. N Engl J Med 350: 1387-1397, 2004.

14. Curb JD, Abbott RD, Rodriguez BL, Sakkinen P, Popper JS, Yano K and Tracy RP: C-reation protein and the future risk of thromboembolic stroke in healthy men. Circulation 107: 2016-2020, 2003.

15. Schupp M,Janke J, Clasen R, UngerT and Kintscher U: Angiotensin type-1 receptor blockers induce peroxisome proliferator-activated receptor-gamma activity. Circulation 109: 2054-2057, 2004.

16. Padda RS, Shi Y, Lo CS, Zhang SL and Chan JS: Angiotensin-(1-7): A novel peptide to treat hypertension and nephropathy in diabetes? J Diabetes Metab 6: 1-6, 2015.

17. Padda RS, Gkouvatsos K, Guido M, Mui J, Vali H and Pantopoulos K: A high-fat diet modulates iron metabolism but does not promote liver fibrosis in hemochromatotic $\mathrm{Hjv}^{-/}$mice.Am J Physiol Gastrointest Liver Physiol 308: G251-G261, 2015.

18. Kurz KD, Main BW and Sandusky GE: Rat model of arterial thrombosis induced by ferric chloride. Thromb Res 60: 269-280, 1990.

19. Chen WQ, Zhang Y, Zhang M, Ji XP, Yin Y and Zhu YF: Establishing an animal model of unstable atherosclerotic plaques. Chin Med J (Engl) 117: 1293-1298, 2004.

20. Chen WQ, Zhang L, Liu YF, Chen L, Ji XP, Zhang M, Zhao YX, Yao GH, Zhang C, Wang XL, et al: Prediction of atherosclerotic plaque ruptures with high-frequency ultrasound imaging and serum inflammatory markers. Am J Physiol Heart Circ Physiol 293: H2836-H2844, 2007.

21. Cormode DP, Skajaa T, Fayad ZA and Mulder WJ: Nanotechnology in medical imaging: Probe design and applications. Arterioscler Thromb Vasc Biol 29: 992-1000, 2009.

22. Cormode DP, Briley-Saebo KC, Mulder WJ, Aguinaldo JG, Barazza A, Ma Y, Fisher EA and Fayad ZA: An ApoA-Imimetic peptide high-density-lipoprotein-based MRI contrastagent for atherosclerotic plaque composition detection. Small 4: 1437-1444, 2008.

23. Clarke SE, Beletsky V, Hammond RR, Hegele RA and Rutt BK: Validation of automatically classified magnetic resonance images for carotid plaque compositional analysis. Stroke 37: 93-97, 2006.

24. Drakopoulou M, Toutouzas K, Michelongona A, Tousoulis D and Stefanadis C: Vulnerable plaque and inflammation: Potential clinical strategies. Curr Pharm Des 17: 4190-4209, 2011.

25. Li D, Chen H, Romeo F, Sawamura T, Saldeen T and Mehta JL: Statins modulate oxidized low-density lipoprotein-mediated adhesion molecule expression in human coronary artery endothelial cells: Role of LOX-1. J Pharmacol Exp Ther 302: 601-605, 2002.

26. Li DY, Chen HJ and Mehta JL: Statins inhibit oxidized-LDLmedated LOX-1 expression, uptake of oxidized-LDL and reduction in PKB phosphorylation. Cardiovasc Res 52: 130-135, 2001. 